\title{
Millimetre-wave imaging with reduced number of degrees of freedom in array signal processing: concepts for enhanced angular resolution
}

\author{
G. Kotyrba and H. Chaloupka
}

Fachbereich Elektrotechnik und Informationstechnik, Bergische Universität Wuppertal, Rainer-Gruenter-Str. 21, 42119

Wuppertal, Germany

\begin{abstract}
This paper deals with millimetre-wave imaging systems based on linear receiving arrays and is focused on the issue of angular resolution and coverage for arrays where the number $M$ of ports is smaller than the number $N$ of elements due to forming of subarrays. For a wide angular coverage a trade-off between a narrow beam width and a high side-lobe suppression is shown to occur which requires proper synthesis of the subarray pattern. Two concepts for an enhanced angular resolution are presented. The first uses a combination of a frequency dependent distribution network (frequency scanning) with array signal processing to enhance angular resolution by cost of a reduced range resolution. The second approach takes advantage of relative movements to enhance angular resolution via a combined angular and temporal (Doppler) processing.
\end{abstract}

\section{Introduction}

Active millimetre-wave imaging systems are expected to provide useful functionality in a wide range of applications, e.g. automotive cruise control (Russell,1997) and robotics if the cost for such systems can be kept at an affordable level. Depending on the special type of application the imaging radar system has to meet certain requirements with respect to the maximum range, range resolution and accuracy, the angular field of view (angular coverage), angular resolution and accuracy as well as the update rate. This paper is in particular focused on angular resolution and coverage.

In principle, the angular information can be derived from either a sequential or a simultaneous (monopulse) angular scanning mode. The first approach includes mechanically steered directional antennas as well as phased arrays and switched multi-beam antenna arrays. The latter approach comprises array signal processing (ASP) techniques based on an architecture with parallel receivers and array signal opera-

Correspondence to: G. Kotyrba

(kotyrba@hft.uni-wuppertal.de) tions in the digital domain. In case of a scenario with relative movements between objects the information retrieved from sequential and simultaneous scanning may significantly differ from each other due to temporal fluctuations of the radarcross-sections (RCS) of scattering centres.

In all approaches for estimating the angular distribution of the backscatterers from received data, the angular resolving power is related to the linear size $D$ of the aperture in terms of the free space wavelength $\lambda$. In case of conventional beamforming the angular resolution is on the order of the 3$\mathrm{dB}$ beam width. For a required resolution $\delta \Theta$ the necessary aperture size thus becomes

$D_{\text {conv }} \geq \frac{50 \lambda}{\delta \Theta / \operatorname{deg}}$.

High-resolution approaches such as minimum variance direction estimator (MVDR) and MUSIC are capable of resolving scattering centres within the beam width. However, since for these methods the angular accuracy rapidly degrades with the number of scatterers separated by less than a beam width (Friedlander 1991, Wax 1989, Bresler 1986), only a small fraction of $N-1$ (with $N$ as the array order) scatterers within a beam width can be resolved. Thus, the beam width influences the resolving power of high-resolution approaches as well. The second important parameter is the available number of degrees of freedom $M-1$ in beamforming which corresponds to $M$ antenna ports possessing $M$ mutually orthogonal radiation pattern. Furthermore, $M$ parallel receiver branches are required for ASP systems or $M$ electronic phase-shifters for phased array systems. For a linear array and about 180 degree angular coverage half-wave element spacing is required leading to $N=2 D / \lambda+1$ element ports and therefore to a maximum number of $M_{\max }-1=2 D / \lambda$ degrees of freedom.

Provided cost saving issues at the device level are successfully solved, the operational frequencies for the considered millimetre wave radar systems can be chosen to be about $75 \mathrm{GHz}$ and may even move to values above $100 \mathrm{GHz}$. Consequently, in most applications the implementation of an ar- 


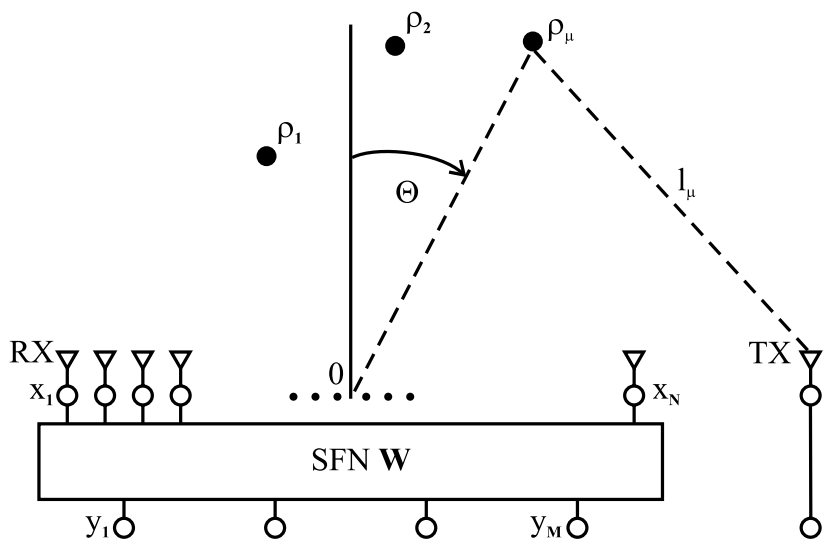

Fig. 1. Considered imaging system with transmitting antenna TX, scattering centers $\rho_{\mu}$, linear receiving array with $N$ elements and subarray forming network (SFN) with $M<N$ output ports.

ray which is according to Eq. (1) sufficiently large does not represent a serious problem anymore but the implementation of $M_{\max }$ receiver channels (or even phase shifters for phased array systems) becomes very costly. It is therefore of interest to exploit less than the available number $M_{\max }-1$ degrees of freedom for beamforming. This is accomplished by employing fixed millimetre wave beamforming networks composed of fixed phase shifters and power dividers to provide from in total $N$ antenna elements $M<M_{\max }=N$ subarrays possessing mutually orthogonal subarray radiation pattern.

This paper aims to discuss the impact of a suboptimal $\left(M<M_{\max }\right)$ number of degrees of freedom onto the angular resolution capabilities and presents concepts which partially allow to overcome performance degradations caused by the reduced number $M$. In Sect. 2 a model and the corresponding notations for the problem under consideration is introduced. Section 3 provides some general results for the system degradations and discusses criteria for an "optimum" choice of the subarrays. In Sect. 4 a novel combination of frequency scanning with array signal processing is proposed which allows to trade-off resolving in range and angle. Section 5 discusses an approach which combines array signal processing with temporal Doppler processing in order to enhance angular resolution.

\section{Representation of the considered problem}

The system to be discussed aims to produce a twodimensional "mm-wave image" of a 3-dimensional scenario composed of various objects. In the used linear model the complex backscattering phenomena are replaced with a set of independent scattering centres. Scattering by a scattering centre can be characterised by a scattering coefficients $\rho_{i}\left(\boldsymbol{u}_{\text {inc }}, f\right)$ with $\left|\rho_{i}\right|^{2}=\sigma_{i}$ representing the radar crosssection. Both quantities depend on the aspect direction $\boldsymbol{u}_{\text {inc }}$, frequency and polarisation. The scalar quantities are understood to be the values which apply to the specific polarisa- tion of the employed TX and RX antennas. The position of scattering centre $i$ is in two dimensions characterised by the azimuth angle $\Theta_{i}$ ("look direction") or the sinus of the look direction $\sin \Theta_{i}=u_{i}$ and the path length $l_{i}$ or corresponding time delay $\tau_{i}=l_{i} / c$ between phase centre of transmission antenna to the scatterer and back to the centre of the receiving antenna.

The following considerations are based on the assumption that a pulse radar with centre frequency $f_{0}$, effective frequency bandwidth $\Delta f$ and pulse repetition frequency $1 / T_{r}$ is used. Furthermore, a bistatic configuration with a single transmission antenna and a linear array as receiving antenna (see, Fig. 1) is considered. Modification of the results to account for other configurations like a monostatic configuration or a multiple-input/ multiple-output (MIMO) configuration are straightforward.

With conventional (no high-resolution approach) processing two different path-lengths can be resolved if the corresponding complex-valued envelopes possess a sufficiently small overlap in time. This leads to a path length resolution of about $\Delta l \approx c / \Delta f$. If a periodically switched time gate (range gate) is allocated to each path length interval of length $\Delta l$ the echos from scattering centres falling into this interval are sampled in time ("snapshots") with a time increment of $T_{r}$. The path lengths can change in time if relative movements between antenna and scattering centres occur. In order to move the corresponding echo from one range gate into the adjacent range gate the path length must change by $\Delta l$. However, path length changes on a much smaller scale lead to phase changes from snapshot to snapshot. A path length change of $c / f_{0}$ leads to a change in phase by $2 \pi$. For $f_{0}=$ $75 \mathrm{Ghz}$ and $\Delta f=1 \mathrm{GHz}$ for example, a $300 \mathrm{~mm}$ path length change are required for a shift into another range gate but only $4 \mathrm{~mm}$ for a phase change of $2 \pi$.

At each instant in time ("snapshot") which corresponds to a considered path length interval (or range gate) a set of $N$ complex amplitudes $\boldsymbol{x}=\left[x_{1}, x_{2}, \ldots, x_{N}\right]^{T}$, with superscript $T$ denoting the transposition, is induced at the $N$ antenna elements. If $M<N$ subarrays are formed via a (ideally) lossless distribution network ("subarray forming network", $\mathrm{SFN})$ the $N$-dimensional vector $\boldsymbol{x}$ is transformed into a $M$ dimensional vector

$\boldsymbol{y}=\mathrm{W} \cdot \boldsymbol{x}$.

$\mathbf{W}$ exhibits $M<N$ rows and $N$ columns and $\mathbf{W} \cdot \mathbf{W}^{H}=1$ holds. In the following sections the properties of the multiport antenna consisting of the $N$ elements without SFN (number of ports $L=N$ ) has to be compared with the multiport antenna build from the $N$-element array in combination with the SFN $(L=M<N)$. For ease of consideration it is assumed that within the field of view in vertical direction the radiation pattern of the considered antennas can be assumed to be constant so that only the dependence on $\Theta$ and therefore on $u=\sin \Theta$ has to be taken into account. In the remaining part of this paper the variable $u$ is used and for convenience referred to as "angle". 
The properties of a general $L$-port antenna are completely described by the steering vector

$\boldsymbol{s}(u)=\left[C_{1}(u), C_{2}(u), \ldots, C_{L}(u)\right]^{T}$

where the functions $C_{1}(u)$ represent the directivity in direction $u$ associated with port $l$. The set of all steering vectors span a $L$-dimensional space $\mathbf{S}_{L}$. Assuming a fictious lossless distribution network connected to this $L$-port antenna and the weights adjusted such that the gain $g$ (or directivity on the lossless case) is maximised towards a given look direction $u_{0}$, one finds for the weights to be given by

$\boldsymbol{w}\left(u_{0}\right)=\boldsymbol{s}\left(u_{0}\right) /\left|\boldsymbol{s}\left(u_{0}\right)\right|$.

Therefore the gain-function becomes

$g\left(u, u_{0}\right)=\boldsymbol{w}^{H}\left(u_{0}\right) \cdot \boldsymbol{s}(u)=$

$\left|\boldsymbol{s}^{H}\left(u_{0}\right) \cdot \boldsymbol{s}(u)\right|^{2} /\left|\boldsymbol{s}\left(u_{0}\right)\right|^{2}$

with the maximum gain given by

$g_{\max }\left(u_{0}\right)=g\left(u_{0}, u_{0}\right)=\left|s\left(u_{0}\right)\right|^{2}$

In order to allow the properties of different multi-port antennas to be compared with each other the functions $g_{\max }\left(u_{0}\right), \Delta U\left(u_{0}\right)$ and $L\left(u_{0}\right)$ can be considered. Function $g_{\max }\left(u_{0}\right)$ characterises the angular coverage of the $L$-port antenna. If a homogeneous coverage of all look angles in the interval $-u_{0, \text { max }}<u_{0}<u_{0, \max }$ is required the condition

$g_{\max }\left(u_{0}\right) \approx \mathrm{const}$ for $-u_{0, \max }<u_{0}<u_{0, \max }$

should be met. The width of the maximum of the gainfunction at $u_{0}$ defines the local beam width which is introduced as

$\Delta u\left(u_{0}\right)=2 \sqrt{g\left(u_{0}, u_{0}\right) / \frac{\mathrm{d}^{2} g}{\mathrm{~d} u^{2}}\left(u=u_{0}, u_{0}\right)}$.

Beside the main maximum the gain-function can possess local maxima (side-lobe) with highest side-lobe at $u_{s l}$. This property is described by

$L\left(u_{0}\right)=g\left(u_{s l}, u_{0}\right) / g\left(u_{0}, u_{0}\right)$.

Measurement of backscattered field from scattering centres in look direction $u_{1}$ to $u_{Q}$ with the scattering amplitudes $\rho_{1}$ to $\rho_{Q}$ are represented by the $L \times L$ covariance matrix

$\mathbf{R}=\left\langle\sum_{v=1}^{Q} \sum_{\mu=1}^{Q} \rho_{v}^{*} \rho_{\mu} s^{*}\left(u_{v}\right) \cdot \boldsymbol{s}^{T}\left(u_{\mu}\right)\right\rangle+P_{\text {noise }} \mathbf{1}$.

In Eq. (10) the scattering coefficients are understood to include the path-loss in wave propagation. $P_{\text {noise }}$ represents noise contribution. The symbol \langle\rangle denotes the mean of covariance matrices which are gained from different snapshots (periodic sampling of range gate with time increment $T_{r}$ ). In case of a scene without relative movement the contributions from different scattering centres have to be considered as correlated. However, if scattering centres possess relative movements, their phase difference varies from snapshot to snapshot so that their contributions become de-correlated leading to

$\mathbf{R}=\sum_{\mu=1}^{Q} \sigma_{\mu} \boldsymbol{s}^{*}\left(u_{\mu}\right) \cdot \boldsymbol{s}^{T}\left(u_{\mu}\right)+P_{\text {noise }} \mathbf{1}$.

The results for the general L-port antenna can now be applied to the array without subarray forming network (SFN) with $L=N$ and to the array with SFN and $L=M<N$. For the linear array with $N$ identical elements with element pattern $\mathbf{C}_{e l}(\Theta, \Phi) \approx \sqrt{g_{0}}$ in the field of view and spacing $a=\lambda / 2$ for 180 degree angular coverage the steering vector becomes

$\boldsymbol{s}_{x}(u)=[1, \exp (j \pi u), \exp (j 2 \pi u), \ldots, \exp (j \pi(N-1) u)]^{T}$
$\exp (-j 0.5(N-1) \pi u) \sqrt{g_{0}}$.

Steering the maximum gain into the look direction $u_{0}$ results in a gain function

$g_{x}\left(u, u_{0}\right)=s_{x}^{H}(u) \cdot s_{x}\left(u_{0}\right)=$

$\frac{g_{e l}}{N} \sin ^{2}\left[\frac{N}{2} \pi\left(u-u_{0}\right)\right] / \sin ^{2}\left[\frac{1}{2} \pi\left(u-u_{0}\right)\right]$

with local (differential) beam width for $N \geq 2$ estimated via

$\Delta u\left(u_{0}\right)=\frac{1}{\pi} \sqrt{\frac{24}{N^{2}-1}}$

and maximum gain

$g_{\max }\left(u_{0}\right)=s_{x}^{H}\left(u_{0}\right) \cdot s_{x}\left(u_{0}\right)=N g_{e l}$.

\section{Impact of reduced number of degrees of freedom on the angular resolution and coverage}

At the output of the subarray forming network (SFN) a $M$-dimensional vector $\boldsymbol{y}$ is available, related to the $N$ dimensional vector $\boldsymbol{x}$ via

$\boldsymbol{y}=\mathbf{W} \cdot \boldsymbol{x}$

Performing ASP to the $M$-dimensional signal vector $\boldsymbol{y}$ with the $M$-dimensional steering vector $s_{y}(u)$ is equivalent with ASP to the $N$-dimensional signal vector $\boldsymbol{x}$ with the $N$ dimensional steering vector

$\boldsymbol{s}_{x}(u)=\mathbf{W}^{T} \cdot \boldsymbol{s}_{y}(u)$.

Equation (17) defines a $M$-dimensional subspace $\mathbf{S}_{y}$ of the $N$-dimensional space $\mathbf{S}_{x}$. Consequently, $\boldsymbol{s}_{x}(u)$ is confined to the subspace $\mathbf{S}_{y} \subset \mathbf{S}_{x}$. Thus, at the output ports of the subarrays only a subset of gain-functions is available. It is therefore of interest to know how the restriction to this subset of gain-functions impacts on the achievable angular resolution and coverage.

Theoretically, there is an infinite number of choices for the formation of subarrays and therefore for the $M \times N$ matrix W. Different $\mathbf{W}$ s result in different steering vector functions $s_{y}(u)$ and in different gain functions $g\left(u, u_{0}\right)$ 


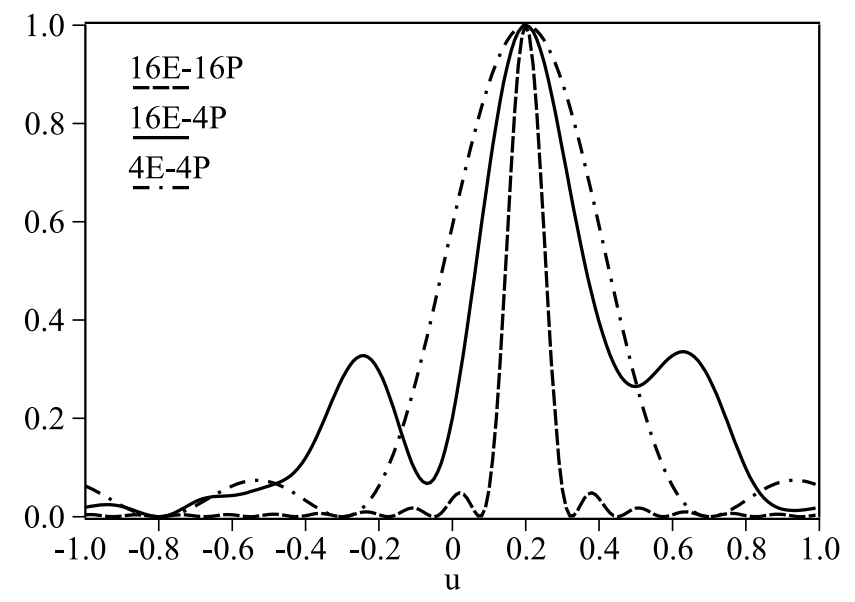

Fig. 2. Normalised gain functions for 3 different antenna configurations which have a angular coverage of $u_{0, \text { max }} \approx 1$ and an element spacing of $\lambda / 2$ in common: Array with $N=M=4$ (4E-4P), array with $N=M=16(16 \mathrm{E}-16 \mathrm{P})$ and array with $N=16$ and $M=4$ (16E-4P) with specifically chosen subarrays.

Hence, there is a need for criteria which allow to select gainfunctions which "optimally" match the requirement for a given imaging problem. The realisation of these optimum gain-functions corresponds to the choice of an optimum matrix $\mathbf{W}$. For the angular coverage requirement

$g_{\max }\left(u_{0}\right) \approx g_{\max , 0}=\mathrm{const}$ in $-u_{0, \max }<u_{0}<u_{0, \max }$

the general restriction

$\int_{-1}^{1} \frac{g\left(u, u_{0}\right)}{g_{\max , 0}} d u \approx 2 \frac{u_{0, \max }}{M}$

can be derived. This means that the area under the normalised gain function for all look angle directions depends on the required angular coverage $u_{0, \max }$ and the number $M$ of subarray ports only. Furthermore, the achievable local differential beam width is limited by the aperture size (excluding superdirective antennas) and therefore by the number $N$ of antenna elements. Eq. (14) may be used as an estimate for the lower limit for $\Delta u$ :

$\Delta u\left(u_{0}\right) \geq \frac{1}{\pi} \sqrt{\frac{24}{N^{2}-1}} \approx \frac{\lambda}{D}$.

The degrees of freedom in synthesising a suitable set of gain functions $g\left(u, u_{0}\right)$ for a given number $M$ of subarrays and a given angular coverage can be deduced from Eqs. (18) and (19). Figure 2 may serve to illustrate the consequences of restrictions (18) and (19). It shows the gain-function (for look-direction $u_{0}=0.2$ as an example) for 3 different array configurations which have an angular coverage of nearly $u_{0, \text { max }} \approx 1$ and a half-wave spacing between antenna elements in common. The first case is an array with $N=4$ elements and $M=N$. The achievable gain function is governed by the area under the gain-function of about 0.5 (Eq. 18) and

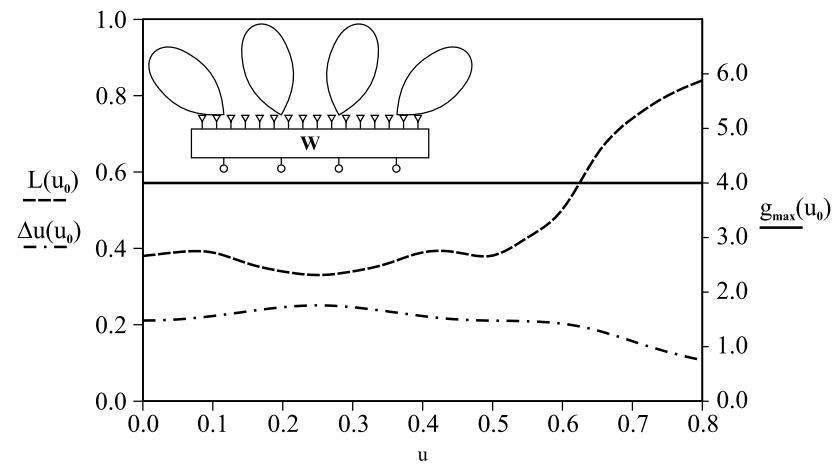

Fig. 3. Dependence of maximum gain $g_{\max }\left(u_{0}\right)$, local beam width $\Delta u\left(u_{0}\right)$ and side-lobe ratio $L\left(u_{0}\right)$ on the steering direction $u_{0}$ for the array with $N=16$ and $M=4$ and specifically chosen subarrays.

the minimum local beam width of about 0.4 (see, Eq. 19). The second case is $M=N=16$. Now the achievable local beam width becomes because of Eq. (19) lower and the area under the gain-curve by a factor of 4 lower. The third case with $N=16$, but $M=4$ belongs to the class of antenna configurations which are of main interest in this paper. Now, the achievable local beam width is governed by $N=16$, but the area under the gain-curve by $M=4$. If the same small beam width as in case 2 is chosen the larger area will lead to 3 side-lobes of equal height as the main lobe. With larger beam width the side-lobes can be made smaller. Case 3 in Fig. 3 represents an example where 4 subarrays were formed from an 16-element array. The main beam directions of these subarray are chosen such that they point into different directions. A minimisation of the variations of the maximum gain $G_{M A X}\left(U_{0}\right)$, the local beam width $\Delta u\left(u_{0}\right)$ and the side-lobe ratio $L\left(u_{0}\right)$ within $\left|u_{0}\right|<u_{0, \max }$ could be a suitable criterium for the choice of the subarrays and therefore of $\mathbf{W}$. Figure 3 illustrates the variations of these 3 quantities with $u_{0}$ in case of the array with $M=4$ and $N=16$ and the particular choice of the subarrays. In this case the subarrays were designed to yield constant maximum gain up to $u_{0}=0.8$.

There is a large number of different concepts for estimating the angular distribution $\sigma(u)$ of the RCS: Conventional (Bartlett estimation)

$\sigma_{1}(u)=\boldsymbol{s}_{y}^{H}(u) \cdot \mathbf{R}_{y} \cdot \boldsymbol{s}_{y}(u)$,

Minimum variance direction estimation (MVDR) as example for a high-resolution approach

$\sigma_{2}(u)=\frac{1}{\boldsymbol{s}_{y}^{H}(u) \cdot \mathbf{R}_{y}^{-1} \cdot \boldsymbol{s}_{y}(u)}$.

In case of the Bartlett estimation the obtained result is significantly improved if the contributions of the different scattering centres are de-correlated, in case of the MVDR decorrelation is a necessary condition. As already explained above, pulse radar yields snapshots of received signal $\mathrm{N}$ dimensional vector $\boldsymbol{x}$ at instances $t=t_{0}, t_{0}+T_{r}, t_{0}+2 T_{r}, \ldots$ 

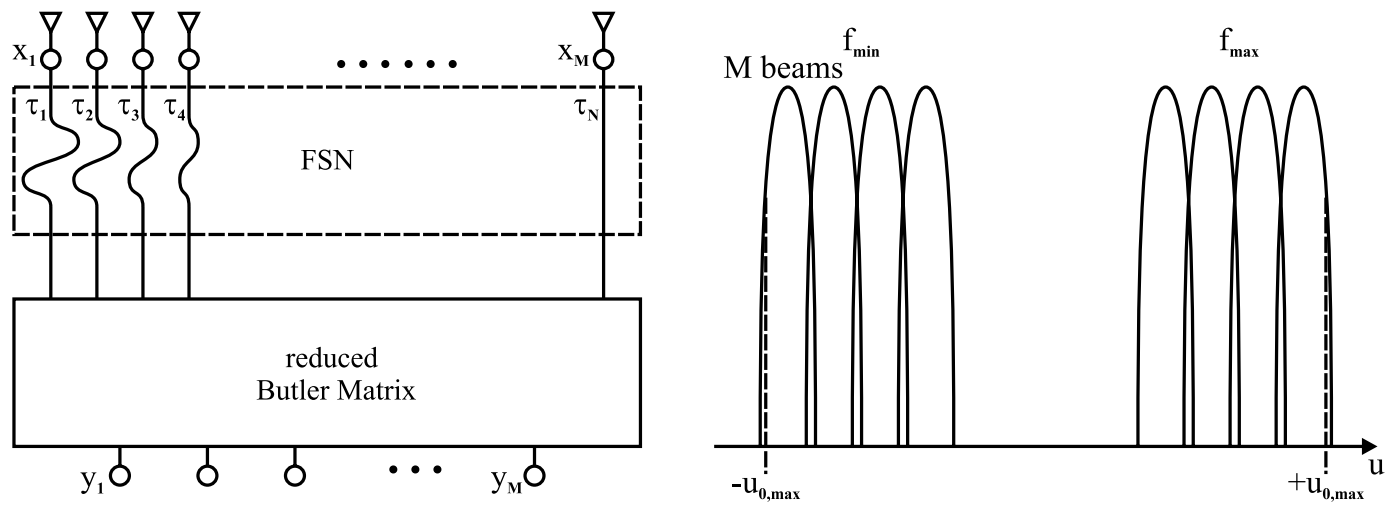

Fig. 4. Combination of frequency scanning with ASP. Left: Configuration with N-dimensional antenna array, frequency scanning network (FSN) and reduced Butler matrix with $M=<N$ output ports. Right: Illustration of frequency scanning for $M=4$ beams.

in time. In case of relative movements, decorrelation is achieved by averaging between different snapshots. For both approaches, Eqs. (20) and (21) angular resolution improves with the local beam width of the gain-function.

\section{Combination of frequency scanning and ASP}

As seen from Eqs. (18) and (19), there is a trade-off for $M<N$ between the demand for a narrow beam width and a low side-lobe ratio of the gain-function on one side and a large angular coverage on the other side. Only if the angular coverage is reduced to

$u_{0, \max } \leq M / N$,

the gain-function can be realised with a beam width and a side-lobe ratio in agreement with the values achieved for $M=N$. This can be accomplished by means of a reduced Butler matrix network. The "full" Butler matrix (BM) for a linear $\mathrm{N}$-element array is a $2 \mathrm{~N}$-port comprising hybrid couplers and fixed phase-shifters with $N$ ports connected to the $N$ antenna elements. The $N$ remaining ports represent the output ports of the BM. As a result of the BM-topology the $N$-dimensional steering vector for the output ports of the full $\mathrm{BM}$ becomes

$\boldsymbol{s}_{\text {Butler }, N}(u)=\left[C_{1}(u), C_{2}(u), \ldots, C_{N}(u)\right]^{T}$ with

$C_{n}(u)=\sqrt{g_{0}} \sin \left[0.5 \pi N\left(u-u_{n}\right)\right] /$

$\sqrt{N} \sin \left[0.5 \pi\left(u-u_{n}\right)\right]$

with $u_{n}=(2 n-N-1) / N$. Hence, each output port corresponds to one of $N$ mutually orthogonal beams and the set of $N$ beams provide a full coverage for $1<u_{0}<1$. For $M<N$ and coverage restricted to angles according to Eq. (22) it is sufficient to have those $M$ of $N$ output ports of the full Butler matrix available which correspond to $M$ adjacent beams with coverage for $u_{0, \max }$ in accordance with Eq. (23). This leads to a reduced BM with $N$ input but only $M$ output ports. The topology of this reduced BM is derived from the topology of the full BM by removing all hybrid couplers and fixed phase-shifters which are exclusively allocated to the $N-M$ ports which do not belong to the $M$ selected ports.

The steering vector in case of the reduced BM follows from that of the full BM if $M-N$ components of $s_{\text {Butler }}(u)$ (see, Eq. 23) are replaced with zeros. The corresponding gain-function is characterised by the same beam width and side-lobe ratio as for the full $\mathrm{BM}$ but the maximum gain $g_{\max }\left(u_{0}\right)$ rapidly drops to low values for $u_{0}>M / N$. A larger angular coverage can be obtained if additionally to the reduced BM a network for frequency scanning (see, Fig. 4) is introduced. This frequency scanning network (FSN) consists of parallel delay lines with different delays $\tau_{n}$ which are inserted between the antenna elements and the BM. Now the radiation pattern becomes frequency-dependent. For a prescribed angular coverage of $u_{0, \max }$ the directional pattern of the linear array at the maximum operational frequency must be shifted by $\Delta u=2\left(u_{\max }-M / N\right)$ relative to the pattern at the minimum operational frequency. As a consequence, the received pulses from the scattering centres become a bandpass-filtered version of the transmitted pulses. The frequency bandwidth of the received pulses is by a factor of $M / N u_{0, \max }$ reduced in comparison to the bandwidth of the transmitted pulses. Therefore the range resolution is reduced by the same factor. Angular resolution is gained by cost of range resolution. Comparing the described combination of ASP with frequency scanning with conventional frequency scanning (only one transceiver channel), the range resolution is improved by a factor of $M$ and additionally high-resolution methods can be applied. Signal processing for angular information requires ASP to be combined with digital filtering in the frequency domain. A severe practical issue associated with this concept is the realisation of relatively large delays in the FSN which tend to introduce dissipative insertion loss.

\section{Combined angular and temporal (Doppler) process- ing}

The $M$-dimensional vectors $\boldsymbol{y}\left(t_{i}\right)$ for the $K=\Delta t / T_{r}$ snapshots taken at $t_{0}, t_{0}+T_{r}, t_{0}+2 T_{r}, \ldots, t_{0}+(K-1) T_{r}$ are 


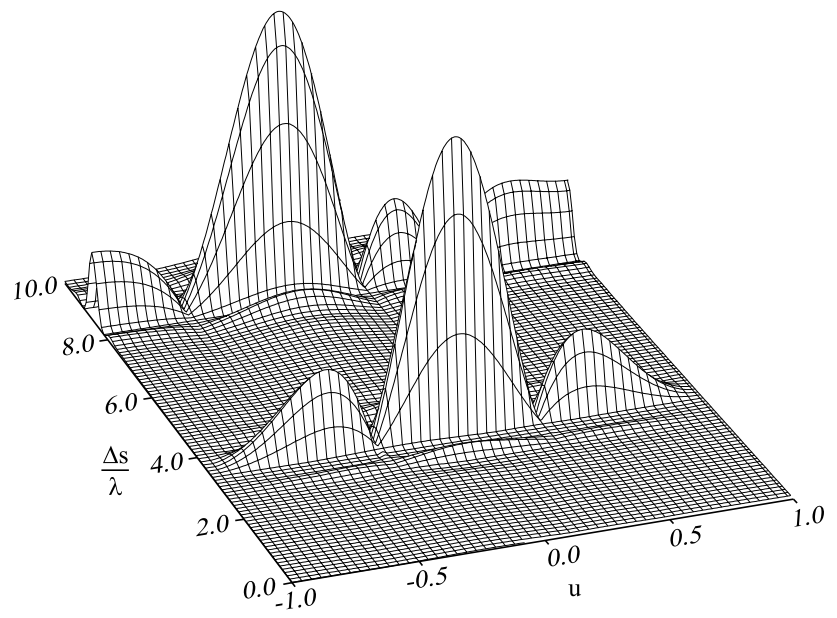

Fig. 5. Resolution of 2 scattering centers in the 2-dimensional angle-Doppler-domain.

taken as input data for a combined processing in angle and Doppler velocity (see e.g. Klemm, 2002). In the simplest processing scheme a 2-dimensional FFT is applied to the $M \times K$ complex values resulting in the 2-dimensional (angle and Doppler) distribution for the scattering centres. This is illustrated by means of an example where backscattered data from 2 scattering centres (separated in angle by $\Delta u=0.34$ ) are assumed to be gained from a linear array with $M=N=4$. Due to the low order (size) of the array the conventional Bartlett estimation (see, Eq. 20) for the angular scatterer distribution is not sufficient to resolve the 2 scattering centres. It is now assumed that within the observation time the changes in pathlengths for both scattering centres differ by about $5.5 \lambda$ (e.g. by $22 \mathrm{~mm}$ in case of $f_{0}=75 \mathrm{GHz}$ ). Figure 5 shows the angle-Doppler distribution as result of the 2D-FFT. Instead of the Doppler velocity the relative shift $\Delta s / \lambda$ is used as a coordinate. Two maxima are clearly separated in this two-dimensional distribution. By applying a non-linear adaptive scaling to this distribution and by projecting back to the coordinate $u$ the angular distribution as shown in Fig. 6 in comparison to the result of the Bartlett estimation is obtained. This demonstrates an improved angular resolution as result of the combined processing in angle and Doppler. With a high-resolution method, e.g. with MVDR (see, Eq. 21), the two scattering centres in this example would also have been resolved without any additional Doppler processing. However, this does not prove that the combined angle-Doppler processing is not superior to the angular processing. If a 2-dimensional high-resolution estimation scheme would be applied to the 2-dimensional angle-Doppler data, a better angular resolution than in case of the one-dimensional high-resolution estimation would be achieved.

The difference between this approach and the syntheticaperture radar (SAR) principle can be explained as follows: In case of SAR there is some knowledge about the direction and velocity of the movement of the scenario relative to the

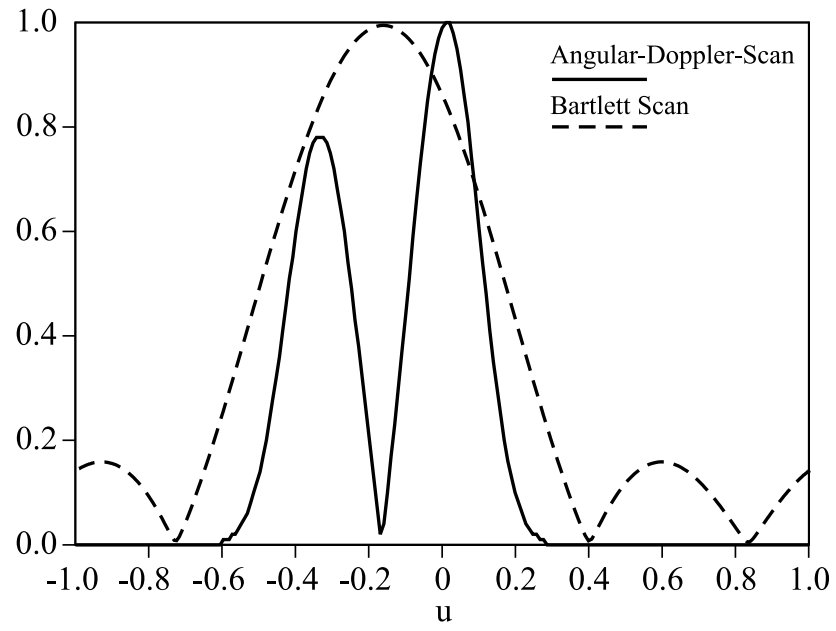

Fig. 6. Improvement of angular resolution by means of angularDoppler-scan in comparison to conventional angular Bartlett scan.

antenna. This enables the different Doppler shifts to be transformed into angular positions and the angular information is gained from Doppler processing. The approach described in this paper does not require any knowledge about direction and velocity of the relative movements. If differential movements of the scattering centres occur an improved angular resolution is achieved but the angular information is retrieved from angular and not from Doppler processing.

\section{References}

Bresler, Y. and Macovski, A.: On the number of signals resolvable by uniform linear array, IEEE Trans. Acoust., Speech, Signal Processing, 34, 1361-1375, 1986.

Friedlander, B. and Weiss, A. J.: On the number of signals whose directions can be estimated by an array, IEEE Trans. Acoust., Speech, Signal Processing, 39, 1686-1689, 1991.

Klemm, R. : Principle of space-time adaptive processing, IEE Radar, Sonar, Navigation and Avionic Series 12, 2002.

Russell, M., Crain, A., Curran, A., Campbell, R., Drubin, C., and Miccioli, W.: Millimeter-wave radar sensor for automotive intelligent cruise control , IEE Trans. Microwave Theory Tech., 45, 2444-2453, 1997.

Wax, M. and Ziskind, I.: On unique localization of multiple sources by passive sensor arrays, IEEE Trans. Acoust., Speech, Signal Processing, 37, 996-1000, 1989. 